\title{
Bioanalysis
}

\section{Paper-based point-of-care testing in disease diagnostics}

\section{"Further advances in the design of paper-based platforms due to new technologies coupled to fabrication optimization will continue to embolden their integration as instruments to understand a wide array of global problems currently impacting mankind."}

First draft submitted: 14 April 2017; Accepted for publication: 5 May 2017; Published online: 23 June 2017

Keywords: disease diagnostics • microfluidic paper-based analytical devices $\bullet$ point-of-care

\section{Statement}

Paper-based microfluidics presents a unique opportunity for diagnosis of various diseases due to their affordability, ease of fabrication, reproducibility, sensitivity and low limits of detection.

Clinical diagnostics play an important role in healthcare in both developed and underdeveloped countries. The incidence rate of communicable and noncommunicable diseases including cancer, cardiovascular and diabetes is much higher in the developing world than in the developed world. According to the WHO, cardiovascular diseases (CVDs) caused about 31\% of global deaths in 2013 with $20 \%$ occurring before the age of 60 [1]. The mortality rate in low-income countries was approximately ten-times higher than that in high-income countries [2]. After CVDs, infectious diseases are a major leading cause of death worldwide [3]. Approximately, 4.3, 1 and 5 million deaths are caused by acute respiratory infection, malaria and tuberculosis and AIDS combined per year in the developing world, respectively [4].

Along with infectious diseases, the cancer rate has also increased in developing countries and now accounts for $65 \%$ or 5.3 million deaths worldwide per year [3]. It is estimated that the annual number of new cancer cases will increase from 14 million in 2014 to 22 million by 2030 . Similar to CVDs and infectious diseases, cancer incidence is highest in developing nations [5].
Roughly, 5.8 billion people worldwide live in low- and middle-resource settings and lack access to expensive medical instruments required for disease detection [6]. Hence, the development of low-cost, simple and portable point-of-care (POC) diagnostic devices is needed to improve patient access to better healthcare delivery with concomitant rapid diagnosis.

POC testing, which goes beyond conventional diagnostic tests in the clinical laboratory setting and that are near patient settings, provides physicians with timely diagnostic information, thereby, allowing them to make better informed decisions regarding diagnosis and treatment [7]. The global POC diagnostics market is expected to increase to US $\$ 27.5$ billion by 2018 and at an estimated compound annual growth rate of $9.3 \%$ from 2013 to 2018 [4].

Currently, there is great interest in using paper-based systems for POC devices due to their simplicity, affordability and ease of use $[8,9]$. Since the seminal work of Whitesides and colleagues in 2007 describing the first microfluidic paper-based analytical devices ( $\mu$ PADs) $[10]$, a myriad of applications in bioanalysis, healthcare and disease screening have been demonstrated. The combination of the capabilities of microfluidic systems with the flexibility of lateral flow test strip technologies has yielded valuable POC diagnostic platforms [11-13]. Paper-based microfluidics presents a unique opportunity for diagnosis

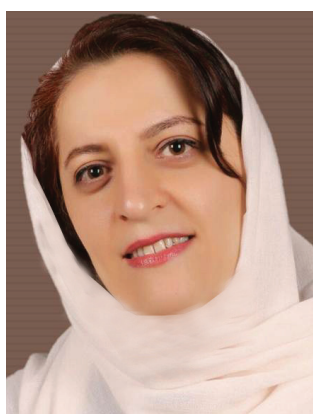

Leila Syedmoradi Biosensor Research Center, Endocrinology \& Metabolism Molecular-Cellular Sciences Institute, Tehran University of Medical Sciences, Tehran, Iran

and

Department of Medical Nanotechnology, School of Advanced Technologies in Medicine, Tehran University of Medical Sciences, Tehran, Iran

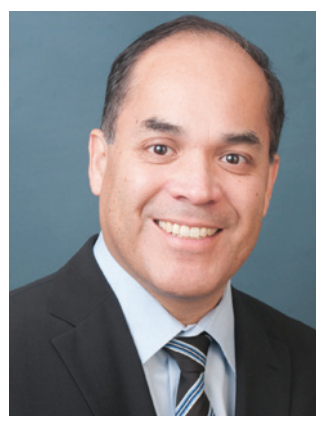

Frank A Gomez Department of Chemistry \& Biochemistry, California State University, Los Angeles, 5151 State University Drive, Los Angeles, CA 90032-8202, USA Tel.: +1 3233432368 Fax: +1 3233436490 fgomez2@calstatela.edu

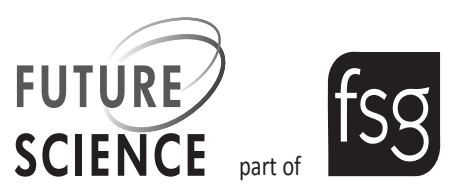


of various diseases due to their affordability, ease of fabrication, reproducibility, sensitivity and low limits of detection. These systems have been applied in the detection of a number of analytes in blood, urine and saliva [6]. Recent work in paper systems has shifted away from the traditional 'single-strip' format, thereby, allowing for a wider range of applications [8,14]. These features make this type of test appropriate for the primary care level and in remote settings where a lack of medical infrastructure may exist.

\section{"Although several studies have focused on using paper-based assays to detect glucose and cancer biomarkers, a few reports have employed this, method for detection of cardiovascular diseases.}

\section{Noncommunicable disease (cancer, CVD \& diabetes)}

Early diagnosis is well known to improve the outcomes of patients, however, conventional approaches are frequently too costly or complex to be implemented in resource-limited settings with little to no medical infrastructure. To overcome this problem, different efforts have been devoted to develop inexpensive, simple paper-based devices as useful alternatives for detection of noncommunicable diseases such as cancer, CVD and diabetes. For example, Wu et al. reported a paper-based electrochemical immunoassay for the simultaneous measurement of four cancer biomarkers in serum samples. The signal amplification was achieved by precipitation of a reduced graphene oxide solution onto the working electrode and through silica nanoparticles as a tracing tag. This strategy provided high-throughput, simple and inexpensive POC immunoassays of cancer biomarkers [15].

When using $\mu$ PADs, it is often difficult to eliminate all nonspecific binding. Liu et al. have described a novel washing strategy in the detection of carcinoembryonic antigen using a ring-oven technique integrated onto the $\mu \mathrm{PAD}$ that washes all nonspecific binding protein from the region of detection ensuring a low background reading [16]. The paper device is similar in size to the ring-oven and yields a LLOD $(0.03 \mathrm{ng} / \mathrm{ml})$ by ELISA. The washing efficiency was greater than that found in traditional techniques. Recently, Zhou et al. constructed a paper dot immune-graphene-gold filtration assay using photothermal properties of graphene/gold nanocomposite for specific detection of MCF-7. Due to the simple, rapid and relatively low detection limit of 600 MCF-7 cells, this method is capable of cancer diagnosis in hospitals [17].

In addition to cancer biomarkers, glucose, the most important analyte to monitor and diagnosis of diabetes, has also been investigated using POC diagnostics.
Gonzalez et al. developed thread/paper-based analytical devices in both flat- and cone-shape platforms to detect glucose via a colorimetric assay. The thread/paper-based analytical devices were fabricated from nylon thread and circular zones of chromatography paper that were previously spotted with glucose. Solutions of glucose oxidase (GOx), horseradish peroxidase (HRP) and potassium iodide were transported to the analysis sites where a yellow color was realized, detected and analyzed [18].

Although several studies have focused on using paperbased assays to detect glucose and cancer biomarkers, a few reports have employed this method for detection of CVDs. Lin et al. developed a simple and inexpensive paper-based device for the detection of C-reactive protein (CRP) using by a latex agglutination test. The paper was initially patterned via the wax-printing method to create three fluidic channels. The wax-printed paper was then modified with different concentration of commercial latex. A blood sample was then added onto the center of each channel to initiate the agglutination reaction with CRP. Finally, CRP levels were determined by serum stain length within the channel [19].

Dong et al. developed a technique for rapid and inexpensive detection of CRP from blood by integrating a paper-based microfluidic immunoassay with a smartphone. The method was based on sandwich immunoassays in which CRP was captured by gold nanoparticle-conjugated anti-CRP antibody. Here, the CRP-bounded particles are captured by antibodies generating a colorimetric signal. The linear detection range of the CRP-chip is up to $2 \mu \mathrm{g} / \mathrm{ml}$ and is capable of detecting $54 \mathrm{ng} / \mathrm{ml}$ within $15 \mathrm{~min}$ [20]. The technique has the potential to improve chronic kidney disease by examining combinatorial multimarker simultaneously and incorporating more sensitive detection technologies.

\section{Communicable disease (infectious diseases)}

The major infectious diseases including HIV, dengue, malaria, tuberculosis and hepatitis $\mathrm{C}$ virus are particularly attractive in the context of healthcare POC diagnostics. Choi et al. constructed a paper-based device by agarose-incorporated lateral flow test strip for detection of dengue virus (the fastest spreading mosquitoborne diseases). This method improved the sensitivity of a modified test strip by approximately tenfold compared with an unmodified strip due to the increased interaction between gold nanoparticle-detector probes and target [21]. Recently, a new type of $\mu$ PAD (the 3D slip-PAD) was developed to detect infectious human norovirus. The assay can be conducted in a single step based on a moveable slip design. A 3D fluidic network is designed by a layered construction of wax-patterned papers, resulting in the attainment of variable fluidic paths. The ability to release and mix minute volumes 
of solution allows for an enhancement in reagents and, therefore, an improvement in sensitivity and detection limits $\left(9.5 \times 10^{4}\right.$ copies $\mathrm{ml}^{-1}$ for human norovirus) [22]

Paper-based devices will continue to emerge as a multiplexable platform for disease diagnostics and particularly in resource-challenged settings where there is frequently limited access to expensive and state-of-the-art instrumentation. These techniques provide a number of advantages including small sample volumes, low cost, flexible properties, ease of integration with other fluidic or analytical devices and compatibility with a plethora of biological systems. The facile fabrication of paperbased devices systematically allows their integration into an array of industries (e.g., agriculture, biomedical, wastewater treatment), thereby making them an almost ideal platform for a number of applications. Further

\section{References}

1 World Health Organizations. Cardiovascular diseases (CVDs). www.who.int/mediacentre/factsheets/fs317/en/

$2 \mathrm{Hu}$ J, Cui X, Gong Y et al. Portable microfluidic and smartphone-based devices for monitoring of cardiovascular diseases at the point of care. Biotechnol. Adv. 34(3), 305-320 (2016).

3 Torre LA, Bray F, Siegel RL, Ferlay J, Lortet-Tieulent J, Jemal A. Global cancer statistics. CA Cancer J. Clin. 65(2) 87-108 (2012).

4 Sharma S, Zapatero-Rodriguez J, Estrela P, O’Kennedy R. Point-of-care diagnostics in low resource settings: present status and future role of microfluidics. Biosensors 5(3), 577-601 (2015).

5 Ferlay J, Soerjomataram I, Dikshit R et al. Cancer incidence and mortality worldwide: sources, methods and major patterns in Globocan 2012. Int. J. Cancer 136(5), E359-E386 (2015).

6 Sher M, Zhuang R, Demirci U, Asghar W. Paper-based analytical devices for clinical diagnosis: recent advances in the fabrication techniques and sensing mechanisms. Expert Rev. Mol. Diagn. 17(4), 351-366 (2017).

7 Gomez FA. The future of microfluidic point-of-care diagnostic devices. Bioanalysis 5(1), 1-3 (2013).

8 Cheung SF, Cheng SK, Kamei DT. Paper-based systems for point-of-vare biosensing. J. Lab. Autom. 20(4), 316-333 (2015).

9 Yetisen AK, Akram MS, Lowe CR. Paper-based microfluidic point-of-care diagnostic devices. Lab. Chip 13(12), 2210-2251 (2013).

10 Martinez AW, Phillips ST, Butte MJ, Whitesides GM. Patterned paper as a platform for inexpensive, low-volume, portable bioassays. Angew. Chem. Int. Ed. Engl. 46(8), 1318-1320 (2007).

11 Syedmoradi L, Daneshpour M, Alvandipour M, Gomez FA, Hajghassem H, Omidfar K. Point of care testing: the impact of nanotechnology. Biosens. Bioelectron. 87, 373-387 (2017). advances in the design of paper-based platforms due to new technologies coupled to fabrication optimization will continue to embolden their integration as instruments to understand a wide array of global problems currently impacting mankind.

\section{Financial \& competing interests disclosure}

The authors have no relevant affiliations or financial involvement with any organization or entity with a financial interest in or financial conflict with the subject matter or materials discussed in the manuscript. This includes employment, consultancies, honoraria, stock ownership or options, expert testimony, grants or patents received or pending, or royalties.

No writing assistance was utilized in the production of this manuscript.

12 Ge S, Zhang L, Yu J. Paper-based microfluidic devices in bioanalysis: how far have we come? Bioanalysis 7(6), 633-636 (2015).

13 Gomez FA. Paper microfluidics in bioanalysis. Bioanalysis 6(21), 2911-2914 (2014).

14 Phillips ST, Lewis GG. The expanding role of paper in pointof-care diagnostics. Expert Rev. Mol. Diagn. 14(2), 123-125 (2014).

15 Wu Y, Xue P, Kang Y, Hui KM. Paper-based microfluidic electrochemical immunodevice integrated with nanobioprobes onto graphene film for ultrasensitive multiplexed detection of cancer biomarkers. Anal. Chem. 85(18), 8661-8668 (2013).

16 Liu W, Guo Y, Zhao M, Li H, Zhang Z. Ring-oven washing technique integrated paper-based immunodevice for sensitive detection of cancer biomarker. Anal. Chem. 87(15), 7951-7957 (2015).

17 Zhou J, Zheng Y, Liu J, Bing X, Hua J, Zhang H. A paper-based detection method of cancer cells using the photo-thermal effect of nanocomposite. J. Pharm. Biomed. Anal. 117, 333-337 (2016).

18 Gonzalez A, Estala L, Gaines M, Gomez FA. Mixed thread/ paper-based microfluidic chips as a platform for glucose assays. Electrophoresis 37(12), 1685-1690 (2016).

19 Lin SC, Tseng CY, Lai PL et al. Paper-based CRP monitoring devices. Sci. Rep. 6, 38171 (2016).

20 Dong M, Wu J, Ma Z et al. Rapid and low-cost CRP measurement by integrating a paper-based microfluidic immunoassay with smartphone (CRP-Chip). Sensors 17(4), 684-696 (2017).

21 Choi JR, Yong KW, Tang R et al. Lateral flow assay based on paper-hydrogel hybrid material for sensitive point-ofcare detection of dengue virus. Adv. Healthcare Mater. 6(1), doi:10.1002/adhm.201600920 (2017) (Epub ahead of print).

22 Han KN, Choi JS, Kwon J. Three-dimensional paper-based slip device for one-step point-of-care testing. Sci. Rep. 6, 25710 (2016). 